\title{
Titin-Related Dilated Cardiomyopathy: The Sequence of Events and The Role of Circulating Biomarkers in The Clinical Assessment
}

\section{Przemysław Chmielewski}

National Institute of Cardiology

Grażyna Truszkowska

National Institute of Cardiology

Ilona Kowalik

National Institute of Cardiology

Małgorzata Rydzanicz

Medical University of Warsaw

Ewa Michalak

National Institute of Cardiology

Małgorzata Sobieszczańska-Małek

National Institute of Cardiology

Maria Franaszczyk

National Institute of Cardiology

Piotr Stawiński

Medical University of Warsaw

Małgorzata Stępień-Wojno

National Institute of Cardiology

Artur Oręziak

National Institute of Cardiology

Michał Lewandowski

National Institute of Cardiology

Przemysław Leszek

National Institute of Cardiology

\section{Maria Bilińska}

National Institute of Cardiology

Tomasz Zieliński

National Institute of Cardiology

\section{Rafał Płoski}

Medical University of Warsaw

Zofia T. Bilińska ( $\nabla$ zbilinska@ikard.pl ) 


\section{Research Article}

Keywords: cardiotitinopathy, TTN truncating variants, biomarkers, troponin T, NT-proBNP, malignant ventricular arrhythmia, end-stage heart failure.

Posted Date: July 9th, 2021

DOl: https://doi.org/10.21203/rs.3.rs-692235/v1

License: (c) (1) This work is licensed under a Creative Commons Attribution 4.0 International License. Read Full License 


\section{Abstract}

Titin truncating variants (TTMtv) are known as the leading cause of inherited dilated cardiomyopathy (DCM). Nevertheless, the clinical course is not fully understood and it is unclear whether circulating cardiac biomarkers are helpful in the detection and risk assessment. We sought to assess: 1) early signs of cardiotitinopathy including serum biomarkers: high-sensitivity cardiac troponin T (hs-cTnT) and Nterminal pro-B-type natriuretic peptide (NT-proBNP) in clinically stable patients, and 2) indicators of outcome among TTMtv carriers. Our single-centre cohort included 108 carriers (including 70 DCM patients) from 43 families. Clinical, laboratory and follow-up data were analyzed. The earliest abnormality was left ventricular dysfunction, present in 8,26 and $47 \%$ of patients in the $2 \mathrm{nd}$, 3rd and 4th decade of life, respectively. It was followed by symptoms of heart failure and elevation of NT-proBNP, linked to severe (persistent or transient) left ventricular systolic dysfunction, and later by arrhythmias, preceding both malignant ventricular arrhythmia (MVA) and end-stage heart failure (esHF). Hs-cTnT serum levels were increased in the late stage of the disease only. During the median follow-up of 5.2 years both MVA and esHF occurred in $12 \%$ of TTMtv carriers. In multivariable analysis, NT-proBNP level $\geq 650 \mathrm{pg} / \mathrm{ml}$ was the best predictor of both composite endpoint (MVA and esHF), and of MVA alone. We conclude that assessment of circulating cardiac biomarkers is not useful in the detection of cardiotitinopathies but may be helpful in the risk assessment.

\section{Introduction}

Dilated cardiomyopathy (DCM) is a major cause of heart failure (HF) and has a genetic basis in 40 to $50 \%$ of cases ${ }^{1}$. Titin truncating variants (TTNtv) account for as many as $20-25 \%$ of the genetic background in $\mathrm{DCM}^{2-7}$ of European but not African ancestry ${ }^{8}$. TTMtv are also found in peripartum ${ }^{4,9}$, alcoholic $^{10}$ and chemotherapy-induced cardiomyopathy ${ }^{11}$. Earlier studies showed good response to optimal medical therapy $7,12,13$, impact of mutation location on the course of disease ${ }^{2,6}$ and similar prognosis as in other forms of $\mathrm{DCM}^{2,3}$. Nevertheless, none of the studies involved circulating cardiac biomarkers in baseline characteristics. So, there are no data on their performance in the early stage of the disease or their role as markers of prognosis. The presence of arrhythmia in cardiotitinopathies is not well characterized, either. Recently, the presence of TTNtv was shown to be an important risk factor for clinically significant arrhythmia in DCM patients (pts) ${ }^{14,15}$.

In 2017, we published our data on cardiotitinopathies including characteristics of 16 TTMtv identified in 17 probands and their 29 relatives $^{3}$. Data on the probands were also included in the multicentre study by Akhtar et $\mathrm{al}^{16}$. Since then, we have identified next $29 \mathrm{DCM}$ probands with TTMtv and their 39 relatives. In this study, we sought to assess the clinical characteristics including serum biomarkers, penetrance of abnormal clinical findings and prognostic risk factors in our cohort of TTNtv carriers.

\section{Results}




\subsection{Molecular findings in the study cohort}

We identified 41 different TTNtv in 46 unrelated probands: two variants were shared by two probands each and one was identified in four probands. As a result of analysis of other DCM-related genes, 3 additional variants classified as likely pathogenic according to the American College of Medical Genetics and Genomics (ACMG) criteria ${ }^{17}$ were identified in MYH7, SCN5A, and TNNT2 in 3 probands. Of the 41 TTNtv, 30 were described before in ClinVar, Varsome or HGMD databases and 11 are novel. The identified variants are pathogenic $(n=40)$ or likely pathogenic $(n=1)$ according to ACMG criteria. Two of them are located in the Z-disc (both nonsense), 10 in the l-band (5 nonsense, 4 frameshift and 1 splice-site), 28 in the A-band (12 nonsense and 16 frameshift) and one in the M-band (nonsense). As a result of genetic screening in the families, 68 TTMtv carriers were identified among relatives. Three probands with likely pathogenic variants in other DCM-related genes and their 2 relatives as well as a patient after heart transplantation (HTx) performed at another centre were excluded from the study. The details of the identified variants in TTN and other DCM-related genes are shown in the supplementary Tables S1 and S2.

\subsection{Clinical characteristics of the study population}

The study cohort was composed of 108 subjects: 70 DCM patients (all 43 probands and 27 relatives), 13 subjects with other cardiac abnormalities, labelled as indeterminate cardiomyopathy (xCM) and 25 healthy relatives with no signs of cardiomyopathy. In two women DCM was diagnosed following symptoms of acute HF shortly after a delivery as peripartum cardiomyopathy. Four patients experienced fulminant HF at the onset of their disease; they all underwent left ventricular assist device (LVAD) implantation: as a bridge to urgent HTx in three cases and as a bridge to recovery in one case.

The baseline clinical characteristics of 108 TTNtv carriers is given in the Table 1 and in the supplementary Table S3. In case of three above mentioned patients who could be assessed only in the acute phase of the disease, the data on cardiac biomarker levels and medical therapy were not included in the analysis. The baseline data of the patient who had LVAD implanted as a bridge to recovery were collected during a consequent ambulatory visit. 
Table 1

Baseline clinical characteristics of TTN truncating variant carriers.

\begin{tabular}{|c|c|c|c|c|c|c|c|}
\hline & $\begin{array}{l}\text { All } N= \\
108\end{array}$ & $\begin{array}{l}\text { Probands } \\
\begin{array}{l}N=43 \\
(39.8 \%)\end{array}\end{array}$ & $\begin{array}{l}\text { Relatives } \\
\begin{array}{l}\mathrm{N}=65 \\
(60.2 \%)\end{array}\end{array}$ & p & $\begin{array}{l}\text { Men } \\
\begin{array}{l}N=67 \\
(62.0 \%)\end{array}\end{array}$ & $\begin{array}{l}\text { Women } \\
\begin{array}{l}\mathrm{N}=41 \\
(38.0 \%)\end{array}\end{array}$ & $\mathbf{p}$ \\
\hline Age, years & $\begin{array}{l}39.7 \pm \\
15.5\end{array}$ & $\begin{array}{l}40.1 \pm \\
13.5\end{array}$ & $\begin{array}{l}39.5 \pm \\
16.9\end{array}$ & 0.832 & $\begin{array}{l}37.4 \pm \\
13.8\end{array}$ & $\begin{array}{l}43.6 \pm \\
17.6\end{array}$ & 0.044 \\
\hline Men & $\begin{array}{l}67 \\
(62.0 \%)\end{array}$ & $\begin{array}{l}33 \\
(76.7 \%)\end{array}$ & $\begin{array}{l}34 \\
(52.3 \%)\end{array}$ & 0.010 & & & \\
\hline Probands & & & & & $\begin{array}{l}33 \\
(49.2 \%)\end{array}$ & $\begin{array}{l}10 \\
(24.3 \%)\end{array}$ & 0.010 \\
\hline \multicolumn{8}{|l|}{ Symptoms } \\
\hline Heart failure & $\begin{array}{l}54 \\
(50.0 \%)\end{array}$ & $\begin{array}{l}39 \\
(90.7 \%)\end{array}$ & $\begin{array}{l}15 \\
(23.1 \%)\end{array}$ & <. 001 & $\begin{array}{l}39 \\
(58.2 \%)\end{array}$ & $\begin{array}{l}15 \\
(36.6 \%)\end{array}$ & 0.015 \\
\hline NYHA class $\geq 3$ & $\begin{array}{l}14 \\
(13.0 \%)\end{array}$ & $9(20.9 \%)$ & $5(7.7 \%)$ & 0.045 & $\begin{array}{l}11 \\
(16.4 \%)\end{array}$ & $3(7.3 \%)$ & 0.172 \\
\hline $\begin{array}{l}\text { Family history of } \\
\mathrm{SCD}<50 \text { yrs }\end{array}$ & $\begin{array}{l}28 \\
(25.7 \%)\end{array}$ & $7(16.3 \%)$ & $\begin{array}{l}21 \\
(31.8 \%)\end{array}$ & 0.063 & $\begin{array}{l}16 \\
(23.9 \%)\end{array}$ & $\begin{array}{l}12 \\
(29.3 \%)\end{array}$ & 0.535 \\
\hline \multicolumn{8}{|l|}{$\begin{array}{l}\text { Arrhythmias and } \\
\text { CCD }\end{array}$} \\
\hline Atrial arrhythmias & $\begin{array}{l}25 \\
(23.1 \%)\end{array}$ & $\begin{array}{l}15 \\
(34.9 \%)\end{array}$ & $\begin{array}{l}10 \\
(15.4 \%)\end{array}$ & 0.019 & $\begin{array}{l}20 \\
(29.8 \%)\end{array}$ & $\begin{array}{l}5 \\
(12.2 \%)\end{array}$ & 0.035 \\
\hline nsVT $(n=106)$ & $\begin{array}{l}42 \\
(39.6 \%)\end{array}$ & $\begin{array}{l}26 \\
(60.5 \%)\end{array}$ & $\begin{array}{l}16 \\
(25.4 \%)\end{array}$ & ¿. 001 & $\begin{array}{l}28 \\
(42.4 \%)\end{array}$ & $\begin{array}{l}14 \\
(35.0 \%)\end{array}$ & 0.449 \\
\hline LBBB & $\begin{array}{l}11 \\
(10.2 \%)\end{array}$ & $7(16.3 \%)$ & $4(6.1 \%)$ & 0.110 & $\begin{array}{l}9 \\
(13.4 \%)\end{array}$ & $2(4.9 \%)$ & 0.200 \\
\hline $\begin{array}{l}\text { AV block ( } \geq 1 \mathrm{st} \\
\text { degree) }\end{array}$ & $\begin{array}{l}15 \\
(13.9 \%)\end{array}$ & $8(18.6 \%)$ & $\begin{array}{l}7 \\
(10.8 \%)\end{array}$ & 0.249 & $\begin{array}{l}11 \\
(16.4 \%)\end{array}$ & $4(9.8 \%)$ & 0.331 \\
\hline \multicolumn{8}{|l|}{ Echocardiography } \\
\hline LVEF $<50 \%$ & $\begin{array}{l}66 \\
(61.1 \%)\end{array}$ & $\begin{array}{l}40 \\
(93.0 \%)\end{array}$ & $\begin{array}{l}26 \\
(40.0 \%)\end{array}$ & $\hat{0} .001$ & $\begin{array}{l}52 \\
(77.6 \%)\end{array}$ & $\begin{array}{l}14 \\
(34.1 \%)\end{array}$ & $<.001$ \\
\hline LVEF, \% & $\begin{array}{l}43.5 \pm \\
13.8\end{array}$ & $\begin{array}{l}34.7 \pm \\
10.6\end{array}$ & $\begin{array}{l}49.3 \pm \\
12.6\end{array}$ & ¿. 001 & $\begin{array}{l}39.8 \pm \\
12.5\end{array}$ & $\begin{array}{l}49.6 \pm \\
13.9\end{array}$ & $\dot{0} 001$ \\
\hline LVEDD, mm & $\begin{array}{l}58.3 \pm \\
9.6\end{array}$ & $64.2 \pm 7.6$ & $\begin{array}{l}54.4 \pm \\
8.9\end{array}$ & $\hat{0.001}$ & $\begin{array}{l}61.2 \pm \\
9.0\end{array}$ & $\begin{array}{l}53.6 \pm \\
9.0\end{array}$ & $\hat{0} .001$ \\
\hline LAs, $\mathrm{mm}(\mathrm{n}=103)$ & $\begin{array}{l}40.6 \pm \\
8.1\end{array}$ & $44.9 \pm 7.2$ & $\begin{array}{l}38.1 \pm \\
7.5\end{array}$ & <. 001 & $\begin{array}{l}43.2 \pm \\
7.9\end{array}$ & $\begin{array}{l}36.2 \pm \\
6.5\end{array}$ & $\hat{0.001}$ \\
\hline
\end{tabular}




\begin{tabular}{|c|c|c|c|c|c|c|c|}
\hline & \multirow{2}{*}{$\begin{array}{l}\text { All } N= \\
108\end{array}$} & Probands & Relatives & \multirow[t]{2}{*}{ p } & Men & Women & \multirow[t]{2}{*}{ p } \\
\hline & & $\begin{array}{l}N=43 \\
(39.8 \%)\end{array}$ & $\begin{array}{l}N=65 \\
(60.2 \%)\end{array}$ & & $\begin{array}{l}N=67 \\
(62.0 \%)\end{array}$ & $\begin{array}{l}N=41 \\
(38.0 \%)\end{array}$ & \\
\hline DCM & $\begin{array}{l}70 \\
(64.8 \%)\end{array}$ & $43(100 \%)$ & $\begin{array}{l}27 \\
(41.5 \%)\end{array}$ & \multirow[t]{3}{*}{$\hat{0} 001$} & $\begin{array}{l}55 \\
(82.1 \%)\end{array}$ & $\begin{array}{l}15 \\
(36.6 \%)\end{array}$ & \multirow[t]{3}{*}{$\begin{array}{l}<.001 \\
0.00\end{array}$} \\
\hline indeterminate CM & $\begin{array}{l}13 \\
(12.0 \%)\end{array}$ & $0(0 \%)$ & $\begin{array}{l}13 \\
(20.0 \%)\end{array}$ & & $\begin{array}{l}7 \\
(10.4 \%)\end{array}$ & $\begin{array}{l}6 \\
(14.6 \%)\end{array}$ & \\
\hline no $\mathrm{CM}$ & $\begin{array}{l}25 \\
(23.2 \%)\end{array}$ & $0(0 \%)$ & $\begin{array}{l}25 \\
(38.5 \%)\end{array}$ & & $5(7.5 \%)$ & $\begin{array}{l}20 \\
(48.8 \%)\end{array}$ & \\
\hline \multicolumn{8}{|l|}{$\begin{array}{l}\text { Biomarkers in } \\
\text { stable phase }\end{array}$} \\
\hline $\begin{array}{l}\text { hs-cTnT, ng/l ( } \mathrm{n}= \\
90)\end{array}$ & $\begin{array}{l}4.4[2.9 ; \\
8.6]\end{array}$ & $\begin{array}{l}7.1[4.1 ; \\
9.4]\end{array}$ & $\begin{array}{l}3.6[2.9 ; \\
7.1]\end{array}$ & 0.003 & $\begin{array}{l}6.4[3.1 ; \\
9.2]\end{array}$ & $\begin{array}{l}3.6[2.9 ; \\
7.0]\end{array}$ & 0.0498 \\
\hline hs-cTnT > 14 ng/l & $\begin{array}{l}9 \\
(10.3 \%)\end{array}$ & $4(11.8 \%)$ & $5(9.4 \%)$ & 0.732 & $\begin{array}{l}6 \\
(11.3 \%)\end{array}$ & $3(8.8 \%)$ & 1.00 \\
\hline $\begin{array}{l}\text { NT-proBNP, pg/ml } \\
(\mathrm{n}=69)\end{array}$ & $\begin{array}{l}254[80 \\
1276]\end{array}$ & $\begin{array}{l}631[186 ; \\
2034]\end{array}$ & $\begin{array}{l}125[40 \\
259]\end{array}$ & $\begin{array}{l}<.001 \\
0.01\end{array}$ & $\begin{array}{l}244[76 \\
1214]\end{array}$ & $\begin{array}{l}292[96 \\
2034]\end{array}$ & 0.500 \\
\hline $\begin{array}{l}\text { NT-proBNP > } 125 \\
\mathrm{pg} / \mathrm{ml}\end{array}$ & $\begin{array}{l}47 \\
(69.1 \%)\end{array}$ & $\begin{array}{l}32 \\
(84.2 \%)\end{array}$ & $\begin{array}{l}15 \\
(50.0 \%)\end{array}$ & 0.002 & $\begin{array}{l}34 \\
(68.0 \%)\end{array}$ & $\begin{array}{l}13 \\
(72.2 \%)\end{array}$ & 0.739 \\
\hline $\begin{array}{l}\text { NT-proBNP > } 650 \\
\mathrm{pg} / \mathrm{ml}\end{array}$ & $\begin{array}{l}27 \\
(39.7 \%)\end{array}$ & $\begin{array}{l}20 \\
(52.6 \%)\end{array}$ & $\begin{array}{l}7 \\
(23.3 \%)\end{array}$ & 0.014 & $\begin{array}{l}19 \\
(38.0 \%)\end{array}$ & 8 & 0.632 \\
\hline \multicolumn{8}{|l|}{$\begin{array}{l}\text { Implantable } \\
\text { devices }\end{array}$} \\
\hline $\begin{array}{l}\text { PM for } \\
\text { bradyarrhythmias }\end{array}$ & $5(4.6 \%)$ & $3(7.0 \%)$ & $2(3.1 \%)$ & 0.385 & $2(4.9 \%)$ & $3(4.5 \%)$ & 1.000 \\
\hline CRT-D & $2(1.8 \%)$ & $2(4.6 \%)$ & $0(0 \%)$ & 0.156 & $2(3.0 \%)$ & $0(0 \%)$ & 0.525 \\
\hline $\begin{array}{l}\text { ICD in secondary } \\
\text { prophylaxis }\end{array}$ & $1(0.9 \%)$ & $0(0 \%)$ & $1(0.9 \%)$ & 1.000 & $0(0 \%)$ & $1(2.4 \%)$ & 0.380 \\
\hline ICD/CRT-D & $\begin{array}{l}14 \\
(13.0 \%)\end{array}$ & $\begin{array}{l}11 \\
(25.6 \%)\end{array}$ & $3(4.6 \%)$ & 0.001 & $\begin{array}{l}10 \\
(14.9 \%)\end{array}$ & $4(9.8 \%)$ & 0.438 \\
\hline
\end{tabular}

Legend: Number of subjects is expressed as $\mathrm{n}(\%)$. Continuous variables are shown as mean \pm standard deviation or median and quartiles [Q1:25th- Q2:75th percentiles]. AV block, atrioventricular block; CCD, cardiac conduction defect; $C M$, cardiomyopathy; CRT-D, cardiac resynchronization therapy defibrillator; DCM, dilated cardiomyopathy; HF, heart failure; hs-cTnT, high-sensitivity cardiac troponin T serum concentration; ICD, implantable cardioverter defibrillator; LAs, left atrial systolic dimension; LBBB, left bundle branch block; LVEDD, left ventricular end-diastolic dimension; LVEF, left ventricular ejection fraction; nsVT, non-sustained ventricular tachycardia; NT-proBNP, N-terminal pro-B-type natriuretic peptide 
serum concentration; NYHA class, New York Heart Association functional class; PM, pacemaker; SCD, sudden cardiac death.

Compared between probands and relatives, we found no significant difference in age, suggesting incomplete penetrance and mild course of cardiotitinopathy in some carriers. Indeed, among evaluated relatives there were not only 14 probands' offspring and 23 siblings, but also 16 parents and a grandmother. Understandably, HF in probands was more common ( $91 \%$ vs $23 \%$ in relatives, $\mathrm{p}<0.001)$, and more advanced in terms of symptoms, as well as echocardiographic and biochemical exponents: New York Heart Association (NYHA) class $\geq 3: 21$ vs 8\%, left ventricular ejection fraction (LVEF): 35 vs 49\%, N-terminal pro-B-type natriuretic peptide (NT-proBNP) serum concentration: 631 vs $125 \mathrm{pg} / \mathrm{ml}$, respectively). Probands had more arrhythmias, both atrial (AA) and ventricular (VA): 35 vs $14 \%$ and 61 vs $25 \%$, respectively, but did not differ significantly with regard to cardiac conduction defects (CCD). Implantable cardioverter defibrillators (ICD) had been implanted more often in probands (30 vs $5 \%$ ).

Median high-sensitivity cardiac troponin T (hs-cTnT) concentration measured in stable condition was higher in probands but at a low level (7.1 vs $3.6 \mathrm{ng} / \mathrm{l}$, respectively).

There were more probands among male carriers (49 vs $24 \%$ ). It may account partially for higher prevalence of DCM and HF in male vs female patients ( 82 vs $37 \%$ and 58 vs $37 \%$, respectively) and for more demonstrable echocardiographic abnormalities (e.g. LVEF: 40 vs $50 \%$, respectively). AA were more frequent and hs-cTnT concentration was higher in male patients ( $30 \mathrm{vs} 12 \%$ and $6.4 \mathrm{vs} 3.6 \mathrm{ng} / \mathrm{l}$, respectively). No significant differences were found with regard to NT-proBNP concentration, VA, CCD or frequency of ICD implantation.

Of note, sudden cardiac death (SCD) under the age of 50 years in family history was found as often as in $26 \%$ of patients.

\subsection{Penetrance of cardiotitinopathy indicators}

Penetrance of cardiac abnormalities in the course of cardiotitinopathy was age-dependent (Fig. 1 and supplementary Table S4). The earliest abnormality was left ventricular dysfunction (LVD), defined as LVEF $<50 \%$ or left ventricular enlargement (LVE) $>112 \%$, and detected in the $2 \mathrm{nd}$, 3rd and 4 th decade of life in $8 \%, 26 \%$ and $47 \%$ of carriers, respectively. It anticipated by approx. 10 years the onset of symptoms of HF, accompanied by NT-proBNP elevation, transient or persistent severe left ventricular systolic dysfunction (LVSD), and VA. AA and atrioventricular block (AVB) appeared late, preceding occurrence of such adverse events as malignant ventricular arrhythmia (MVA) and end-stage heart failure (esHF). Elevated hs-cTnT concentration seems to be an indicator of end-stage phase of cardiotitinopathy.

The rise in frequency of circulating cardiac biomarker concentrations, observed in the 6th and 7th decades of life may be attributable to co-existing conditions, such as atherosclerosis, rather than to cardiotitinopathy itself. 


\subsection{Results of screening in carriers of cardiotitinopathy- causing truncating variants}

Observations on the sequence of occurrence of individual abnormalities may be biased due to the fact that the disease is often detected at an advanced stage with a number of anomalies present. Therefore, we analyzed the penetrance of cardiac abnormalities also in the group of 49 TTMtv carriers who came to our Unit for screening. The diagnosis of DCM was established in 11 of them, however only one patient developed HF during the follow-up. In this group, LVD was detected as the earliest indicator of TTMtV carriership in 21 (43\%) pts (Fig. 2). Of note, in 16\% of pts, LVE was accompanied by LVSD whereas LVE and LVSD were found as isolated deviations in $16 \%$ and $10 \%$ of pts, respectively. NT-proBNP serum concentration was elevated in only $27 \%$ of pts with LVD.

Arrhythmias and CCD were detected less frequently than in the whole cohort, despite repeated ECG and Holter recordings. Interestingly, AA were detected in 4 (8\%) pts, and they preceded LVD in all of them. Also VA in 2 cases and AVB in one case were detected in subjects without LVD.

\subsection{Outcome and risk stratification in cardiotitinopathy}

The median follow-up in the group of 108 TTNtv carriers was 5.2 years [Q1: 2.1, - Q3: 7.9]. During the follow-up period, 13 (12\%) patients developed esHF (Table 2): twelve men and only one woman $(p=0.02)$. Six $(6 \%)$ of these pts died of HF (one of them shortly after HTX and another one after LVAD implantation) and seven (6.5\%) were transplanted successfully (in 4 cases preceded by LVAD implantation). There was one SCD, two patients had sudden cardiac arrest (SCA) with successful resuscitation. Of the $27(25 \%)$ patients with ICD implanted before the baseline visit or during follow-up, $13(48 \%)$ experienced adequate ICD intervention. 
Table 2

Clinical outcomes in the cohort of TTN truncating variant carriers.

\begin{tabular}{|c|c|c|c|c|}
\hline Events during follow-up & $\begin{array}{l}\text { Total } N= \\
108\end{array}$ & $\begin{array}{l}\text { Men } \\
\begin{array}{l}N=67 \\
(62.0 \%)\end{array}\end{array}$ & $\begin{array}{l}\text { Women } \\
\begin{array}{l}\mathrm{N}=41 \\
(38.0 \%)\end{array}\end{array}$ & $\mathbf{P}$ \\
\hline Implantable devices & \multicolumn{4}{|c|}{ (implanted before baseline visit or during follow-up) } \\
\hline ICD in secondary prophylaxis & $2(1.9 \%)$ & $1(1.5 \%)$ & $1(2.4 \%)$ & 1.00 \\
\hline CRT-D & $7(6.5 \%)$ & $6(9.0 \%)$ & $1(2.4 \%)$ & 0.249 \\
\hline ICD/CRT-D implantation & $27(25.0 \%)$ & $21(31.3 \%)$ & $6(14.6 \%)$ & 0.052 \\
\hline $\begin{array}{l}\text { Malignant ventricular arrhythmia, } n= \\
107\end{array}$ & $13(12.1 \%)$ & $9(13.4 \%)$ & $4(10.0 \%)$ & 0.763 \\
\hline Appropriate ICD intervention, $\mathrm{n}=27$ & $13(48.1 \%)$ & $9(42.9 \%)$ & $4(66.7 \%)$ & 0.384 \\
\hline Cardiopulmonary resuscitation, $\mathrm{n}=106$ & $2(1.9 \%)$ & $2(3.0 \%)$ & 0 & 0.530 \\
\hline Sudden cardiac death, $\mathrm{n}=106$ & $1(0.9 \%)$ & 0 & $1(2.6 \%)$ & 0.368 \\
\hline End-stage heart failure, $n=107$ & $13(12.1 \%)$ & $12(17.9 \%)$ & $1(2.5 \%)$ & 0.024 \\
\hline LVAD & $5(4.6 \%)$ & $5(7.3 \%)$ & 0 & 0.155 \\
\hline Heart transplantation & $8(7.4 \%)$ & $8(11.9 \%)$ & 0 & 0.024 \\
\hline HF death, $n=106$ & $6(5.7 \%)$ & $5(7.5 \%)$ & $1(2.6 \%)$ & 0.410 \\
\hline Death & $10(9.3 \%)$ & $7(10.3 \%)$ & $3(7.3 \%)$ & 0.742 \\
\hline
\end{tabular}

Legend: Number of subjects with events is expressed as n (\%). ATP, antitachycardia pacing; CRT-D, cardiac resynchronization therapy defibrillator; HF, heart failure; ICD, implantable cardioverter defibrillator; LVAD, left ventricular assist device.

We examined the influence of pre-specified risk factors on the risk of occurrence of composite endpoint (end-stage HF or MVA) in the whole cohort of 108 TTMtv carriers (Table 3). The univariable analysis suggests possible influence of such factors, as severely reduced LVEF, dilated left atrium, elevated NTproBNP and hs-cTnT, the presence of left bundle branch block (LBBB), non-sustained ventricular tachycardia (nsVT) or AA. In multivariable analysis, NT-proBNP level $\geq 650 \mathrm{pg} / \mathrm{ml}$ was the best predictor of the composite endpoint at 6 years of follow-up (Fig. 3). The model had good discrimination as evidenced by the C-index of 0.842 [ $95 \% \mathrm{Cl}$ : $0.776-0.908$ ]. 
Table 3

Potential risk factors affecting the risk of occurrence of the composite endpoint of malignant ventricular arrhythmia and end-stage heart failure in cardiotitinopathy.

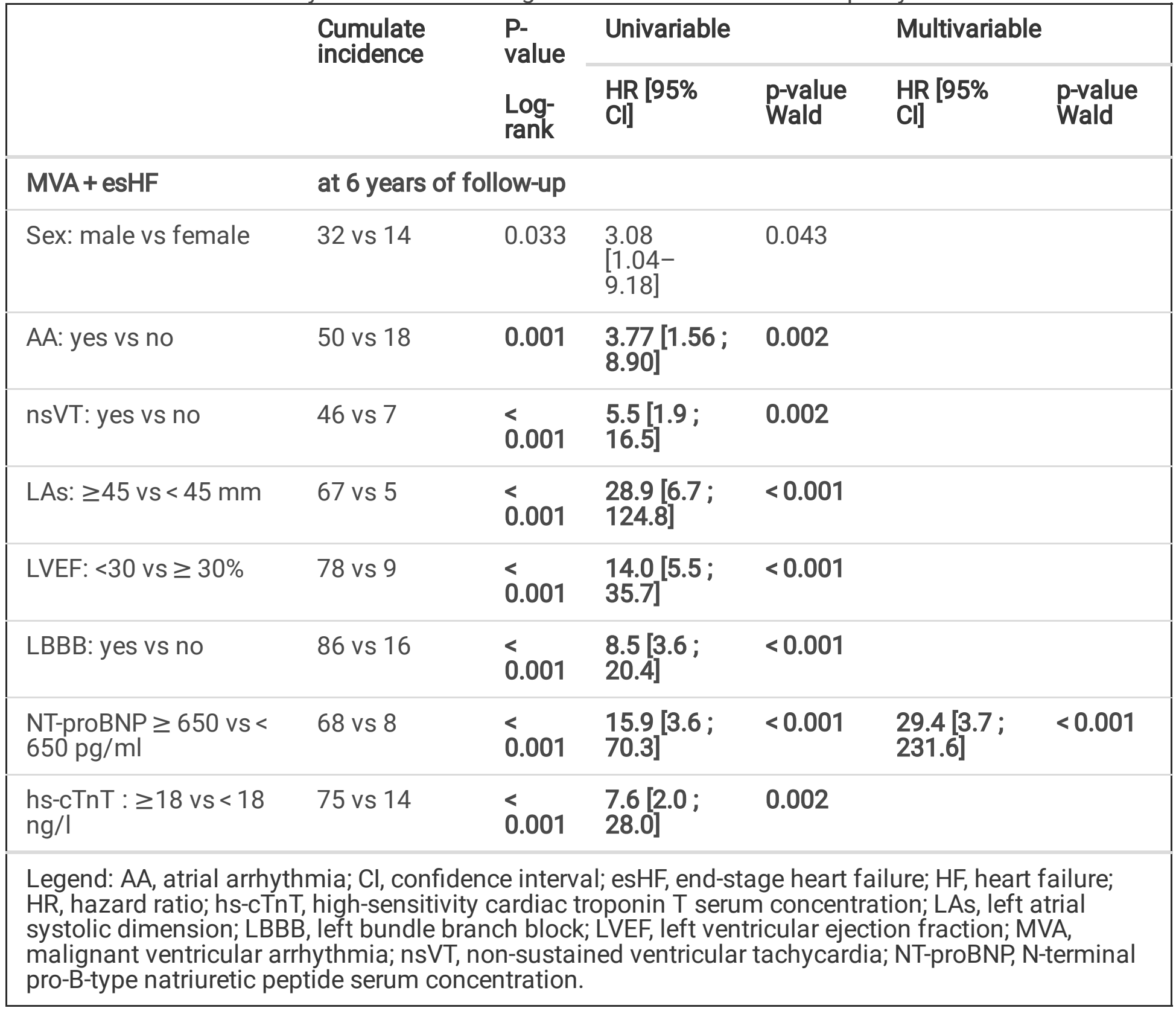

Anticipating life-threatening arrhythmia episodes is even more important as they can be interrupted by ICD interventions. The univariable analysis of MVA events during follow-up in the subgroup of 107 patients with no history of SCA or SVT shows possible influence of such risk factors as severely reduced LVEF, dilated left atrium, elevated NT-proBNP, the presence of LBBB, AA or nsVT but no impact of sex, family history of SCD or elevated hs-cTnT (Table 4). In multivariable analysis, NT-proBNP level $\geq 650$ $\mathrm{pg} / \mathrm{ml}$ was again the best predictor of MVA at 6 years of follow-up (Fig. 4). The model had good discrimination as evidenced by the C-index of 0.787 [95\% Cl: $0.672-0.909]$. 
Table 4

Potential risk factors affecting the risk of occurrence of malignant ventricular arrhythmia in cardiotitinopathy.

\begin{tabular}{|c|c|c|c|c|c|c|}
\hline & \multirow{2}{*}{$\begin{array}{l}\text { Cumulate } \\
\text { incidence }\end{array}$} & \multirow{2}{*}{$\begin{array}{l}\text { P- } \\
\text { value } \\
\text { Log- } \\
\text { rank }\end{array}$} & \multicolumn{2}{|l|}{ Univariable } & \multicolumn{2}{|c|}{ Multivariable } \\
\hline & & & $\mathrm{HR}[95 \% \mathrm{Cl}]$ & $\begin{array}{l}\text { p-value } \\
\text { Wald }\end{array}$ & $\begin{array}{l}\mathrm{HR} \\
\mathrm{Cl}]\end{array}$ & $\begin{array}{l}\text { p-value } \\
\text { Wald }\end{array}$ \\
\hline MVA & \multicolumn{6}{|c|}{ at 6 years of follow-up } \\
\hline Sex: Male vs Female & 21 vs 14 & 0.362 & $\begin{array}{l}1.72[0.52 \\
5.59]\end{array}$ & 0.368 & & \\
\hline $\begin{array}{l}S C D<50 \text { in family: yes } \\
\text { vs no }\end{array}$ & 13 vs 20 & 0.684 & $\begin{array}{l}0.76[0.21 ; \\
2.78]\end{array}$ & 0.685 & & \\
\hline AA: yes vs no & 35 vs 14 & 0.010 & $\begin{array}{l}3.81[1.28 \\
11.36]\end{array}$ & 0.016 & & \\
\hline nsVT: yes vs no* & 39 vs 2 & <. 001 & $\begin{array}{l}12.5[2.2 ; \\
72.7]\end{array}$ & 0.005 & & \\
\hline LAs: $\geq 45 \mathrm{vs}<45 \mathrm{~mm}$ & 52 vs 5 & $\hat{0} .001$ & $\begin{array}{l}16.9[3.7 ; \\
77.4]\end{array}$ & $<0.001$ & & \\
\hline LVEF: $<30 \%$ vs $\geq 30 \%$ & 65 vs 8 & <. 001 & $\begin{array}{l}9.9[3.2- \\
30.2]\end{array}$ & $<0.001$ & & \\
\hline LBBB: yes vs no* & 83 vs 10 & $\hat{0} .001$ & $\begin{array}{l}14.6[4.8 ; \\
44.3]\end{array}$ & $<0.001$ & & \\
\hline $\begin{array}{l}\text { NT-proBNP } \geq 650 \text { vs }< \\
650 \mathrm{pg} / \mathrm{ml}\end{array}$ & 59 vs 8 & $\dot{0} 001$ & $\begin{array}{l}11.7[2.6- \\
53.6]\end{array}$ & 0.002 & $\begin{array}{l}\text { 11.0 [2.3; } \\
53.2]\end{array}$ & 0.003 \\
\hline $\begin{array}{l}\text { hs-cTnT: } \geq 14 \text { vs }<14 \\
\text { ng/l }\end{array}$ & 25 vs 13 & 0.823 & $\begin{array}{l}1.27[0.16 ; \\
10.2]\end{array}$ & 0.823 & & \\
\hline
\end{tabular}

Legend: $\mathrm{AA}$, atrial arrhythmia; $\mathrm{Cl}$, confidence interval; $\mathrm{HF}$, heart failure; $\mathrm{HR}$, hazard ratio; hs-cTnT, highsensitivity cardiac troponin T serum concentration; LAs, left atrial systolic dimention; LBBB, left bundle branch block; LVEF, left ventricular ejection fraction; MVA, malignant ventricular arrhythmia; nsVT, nonsustained ventricular tachycardia; NT-proBNP, N-terminal pro-B-type natriuretic peptide serum concentration; SCD, sudden cardiac death; *, Firth's correction.

\section{Discussion}

A major finding of the study is that the earliest marker of the carrier status in TTNtv-related DCM is left ventricular dysfunction defined as LVEF $<50 \%$ or LVE $>112 \%$ which preceded the development of overt HF and transient or persistent severe LVSD by approximately 10 years which, in turn, was followed by a variety of arrhythmia, both ventricular and atrial, as well as AVB. Of note, among screened relatives $(n=$ 49) isolated LVE with normal LVEF was the first sign of cardiotitinopathy in $16 \%$ of subjects, reduced 
LVEF without LVE in $10 \%$, and both abnormalities were detected simultaneously in $16 \%$ of subjects. LVE is known as the first sign of early $D_{C M}{ }^{18-20}$. TTNtv were also associated with eccentric cardiac remodeling in analysis of cardiac magnetic resonance in healthy humans ${ }^{21}$. This is in agreement with a proposal of a new definition of DCM which recognizes 3 forms of preclinical phase of DCM, including isolated left ventricular dilation ${ }^{22}$. However, in a zebrafish model, serial echocardiography showed significant LVEF reduction in ttnatv/+ vs ttnat/+ zebrafish preceding LVE by 3-6 months ${ }^{23}$. Furthermore, comprehensive genomics-first studies on the impact of TTNtv on cardiac phenotype by Haggerty et al. ${ }^{8}$ and Pirruccello et al. ${ }^{24}$ showed that TTMtv carriers are characterized by lower LVEF but not larger left ventricular (LV) diastolic dimensions or volumes. Our DCM-dedicated study shows that both LVE and LVSD may be the first detectable abnormality.

To the best of our knowledge, circulating cardiac biomarkers in relation to either the disease penetrance or prognosis have not been reported in cardiotitinopathies. The role of circulating cardiac biomarkers in the detection of $\mathrm{HF}$ is widely recognized ${ }^{25}$. In community based studies, multiple cardiac biomarkers are detectable in ambulatory individuals and add prognostic value to standard risk factors for predicting mortality, overall cardiovascular events, and $\mathrm{HF}^{26,27}$. However, little is known about the significance of circulating biomarkers in early stage of DCM in humans ${ }^{26,28}$. We showed recently that elevated hs-cTnT is the earliest marker of the carrier status in cardiolaminopathies ${ }^{29}$ and might be a "red flag" in asymptomatic or mildly symptomatic carriers.

Our data show that determination of widely available serum biomarkers cannot replace echocardiography in detection of affected TTNtv carriers. NT-proBNP serum level is rarely elevated in subjects with mildly reduced LVEF or isolated LVE and it usually exceeds the normal range when HF symptoms and advanced LVSD are present. In contrast to cardiolaminopathies, elevated hs-cTnT concentration seems to be an indicator of end-stage phase of cardiotitinopathies.

In our cohort, AA was found during baseline evaluation in $23 \%$ of TTNtv carriers and VA in $40 \%$. The frequencies are lower than in the largest to date study by Akhtar et al. who found $A A$ in one third and VA in one-half of TTNtv carriers ${ }^{16}$. The difference can be explained by younger population and less advanced LVSD in our study. The timing of the appearance of VA seems similar in both cohorts, in conjunction with LVSD progression.

Authors of several recent papers report significant arrhythmic burden characteristic of TTMtv-associated DCM, most often found in a relatively advanced disease stage. In the study by Corden et al., having a TTNtv was associated with a higher risk of receiving appropriate ICD therapy in the group of 148 DCM patients with implanted ICDs (hazard ratio (HR) 4.9; $<<0.001)^{15}$. In addition, TTNtv was a risk factor for developing new persistent atrial fibrillation $(\mathrm{AF})^{15}$. Tayal et al. found that patients with TTMtv are more likely to have a history of AA or VA at the time of DCM diagnosis ${ }^{14}$. In a Danish study on 115 TTNtvrelated DCM patients with mean LVEF $28 \%$, AF and MVA occurred in $43 \%$ and $23 \%$ of patients, 
respectively ${ }^{30}$. Of note, AF preceded the DCM diagnosis in $16 \%$ of pts, and in $11 \%$ the presenting symptom of DCM was MVA ${ }^{30}$.

In cardiolaminopathies, conduction disease and arrhythmias precede the onset of HF by seven years ${ }^{31-}$ 33. However, $L M N A$ mutations are not a common cause of lone $\mathrm{AF}^{34}$. Arrhythmias are also reported in TTNtv carriers with normal cardiac function. In a case control study that included 2,781 participants with early-onset AF and normal LVEF, and 4,959 controls, there was a statistically significant association between TTNtv and AF (odds ratio, 1.76) ${ }^{35}$. Associations with arrhythmias, including AF, were also observed in the genomics-first study by Haggerty et al., even when conditioning on DCM diagnosis ${ }^{8}$. Among screened relatives in our study, AA or VA was found in 8/50 (16\%) subjects and it was the earliest detected abnormality in $6(12 \%)$ of them. This shows that in the course of cardiotitinopathies various clinical scenarios are possible: arrhythmias appear typically in the late disease stage but they can also precede the DCM diagnosis.

As shown previously by others ${ }^{7,15}$ and us $^{3}$, LBBB is relatively uncommon among cardiotitinopathy patients and therefore CRT-D requirement is less pronounced.

Another major finding of our study was strong, independent association between NT-proBNP level $\geq 650$ $\mathrm{pg} / \mathrm{ml}$ and the occurrence of the composite endpoint of MVA and esHF among TTNtv carriers. Recently several studies defining prognostic factors in cardiotitinopathy have been published, however none of them included impact of circulating biomarkers ${ }^{7,14-16}$.

NT-proBNP serum concentration $\geq 650 \mathrm{pg} / \mathrm{ml}$ was also the best predictor of MVA in our study. Excellent prognostic role of NT-proBNP in patients with HF is widely recognized ${ }^{36}$. The association of raised levels of NT-proBNP and MVA in HF patients was shown previously in general HF cohorts ${ }^{37,38}$. NT-proBNP provides also information regarding the risk of SCD in a community-based population beyond other traditional risk factors ${ }^{39}$.

There is great need for identification of prognostic factors that may help in decision making with regard to ICD therapy. Our study suggests that NT-proBNP, a commonly available circulating biomarker, may be useful in the setting of clinically stable TTMtv carriers.

The hearts of patients with TTNtv and DCM have thinner LV walls and lower indexed LV mass compared to TTMtv-negative controls ${ }^{6}$. It results in higher LV wall stress and release of NT-proBNP, and is associated with increased risk of VA, 65,40 . It explains why NT-proBNP may be a good predictor of both MVA and esHF in TTNtv-related DCM.

Unlike in cardiolaminopathies ${ }^{41}, T T N$ tv-positive DCM patients have midwall replacement fibrosis detected in CMR with similar frequency as TTNtv-negative DCM controls ${ }^{15,40}$ but interstitial fibrosis is found at endomyocardial biopsy significantly more often ${ }^{40}$. We hypothesize that hs-cTnT leakage, detectable from early stages of cardiolaminopathy ${ }^{29}$, may reflect cardiomyocyte death and replacement fibrosis, prevalent 
in $L M N A$-related cardiac disease, but it may be undetactable in interstitial fibrosis, characteristic of cardiotitinopathy. This might explain why hs-cTnT level raises significantly only in the end-stage of TTNtv-related DCM whilst in earlier stages, when interstitial fibrosis and increased risk of life-threatening arrhythmias are already present, it remains within normal range.

Male patients were younger (37 vs 44 years, $p=0.04$ ) and had more advanced HF, similarly as in earlier studies $^{3,16}$. We found more AA among male patients (30 vs $12 \%, p=0.04$ ) but no significant differences with regard to nsVT. In largest to date study on phenotypic characterization of TTNtv carriers male probands had a higher prevalence of AF at baseline ( $20 \mathrm{vs} 5 \%, p=0.01)$, the difference with regard to nsVT was less pronounced (58 vs $42 \%, p=0.046)^{16}$.

As Giudicessi et al stated ${ }^{42}$, the prevalence of TTMtv in the Genome Aggregation Database (1.8\%) is more than 4 times higher than the estimated prevalence of DCM in general population (0.4\%). This underlines the role of TTNtv as susceptibility variants and suggests that strong environmental effects or additional genetic factors contribute to the development of a cardiac phenotype ${ }^{43}$.

In this study we showed that pathogenic or likely pathogenic TTNtv in DCM patients were located in all domains of the gene and had high proportion spliced-in index, as in the study by Akhtar et al. ${ }^{44}$. Early studies $^{2}$, including ours ${ }^{3}$, showed A-band location of TTNtv mutations as more specific for DCM patients. With many more TTNtv identified and more accumulated data, no statistically significant differences in baseline clinical phenotypes or LVSD penetrance associated with TTNtv location across different TTN bands were identified ${ }^{16}$.

\section{Materials And Methods}

\subsection{Study design}

On a prospective basis, genetic testing was offered to all DCM probands in the care of our Unit and cascade screening was offered to all probands' families. All TTNtv, considered to be pathogenic or likely pathogenic according to the American College of Medical Genetics and Genomics (ACMG) criteria ${ }^{16,17}$, were identified between 2012 and 2021.

Data from all persons (probands and relatives) with TTNtv in the care of the National Institute of Cardiology, Warsaw, were retrospectively collected, including baseline clinical information from the first documented visit to the Institute and follow-up data, in particular data for all major cardiovascular events. In case of patients whose first contact to the Institute took place during HF exacerbations the baseline evaluation was moved to latter ambulatory visits whenever possible. We analyzed the baseline data, comprising medical history, clinical examination, 12-lead electrocardiography, two-dimensional Doppler echocardiography, 24-hour Holter ECG monitoring, as well as the serum biomarkers - high-sensitivity cardiac troponin T (hs-cTnT) and N-terminal pro-B-type natriuretic peptide (NT-proBNP), measured during ambulatory visits in patients in stable condition (data on serum biomarkers measured in unstable 
patients were not included). In all probands, coronary angiography or coronary computed tomography angiography was performed.

We sought to examine the order of appearance of abnormal signs in cardiotitinopathy, including elevated circulating biomarkers. We also wanted to evaluate the prognostic value of circulating cardiac biomarker concentrations with regard to occurrence of MVA and esHF during the follow-up period.

The study was funded by the statutory grant 2.56/II/14 from the National Institute of Cardiology, Warsaw, Poland, the grant 2011/01/B/NZ4/03455 from the National Science Centre, Poland, and the recent grant ERA-CVD DETECTIN-HF/2/2017 from the National Centre for Research and Development, Poland. All methods were carried out in accordance with relevant guidelines and regulations All patients and relatives signed written informed consent in accordance with the Declaration of Helsinki. The study received the approval of the Bioethics Committee of the National Institute of Cardiology.

\subsection{Definitions}

Left ventricular enlargement (LVE) was ascertained when the left ventricular end diastolic diameter (LVEDD) exceeded $112 \%$ of the predicted value, corrected for age and body surface area according to Henry's formula, while left ventricular systolic dysfunction (LVSD) was ascertained when left ventricular ejection fraction (LVEF) was $<50 \%$. We used the term left ventricular dysfunction (LVD) when one of the above mentioned criteria was met and the diagnosis of DCM was made when both criteria were met. When no LVE but more distinct LVSD was present (LVEF < 45\%), hypokinetic non-dilated cardiomyopathy (HNDC) was diagnosed; for the purpose of this study, patients with HNDC were included in DCM cohort. When LVEF fell below 35\%, we used the term severe LVSD. In the presence of relevant abnormalities, not sufficient for the diagnosis of DCM/HNDC, such as LVE > 117\%, LVEF $45-49 \%$, cardiac conduction defect (CCD), atrial or ventricular arrhythmias unexplained by other conditions, we used the term indeterminate cardiomyopathy (xCM).

CCD included atrioventricular block (AVB) and left bundle branch block (LBBB). First degree AVB was defined by a PR interval > $200 \mathrm{~ms}$ on standard 12-lead ECG. High degree AVB included type II second degree or third degree AVB. Atrial arrhythmias (AA) included atrial fibrillation (AF), flutter, and paroxysmal atrial tachycardia lasting $\geq 30 \mathrm{~s}$. Ventricular arrhythmias (VA) included venticular ectopy burden of $>$ $500 / 24$ hours on Holter monitoring or non-sustained ventricular tachycardia (nsVT), defined as $\geq 3$ consecutive ventricular beats at $>120 \mathrm{bpm}$ and a duration for $<30$ seconds on Holter monitoring. If the VT lasted over $30 \mathrm{sec}$., it was considered sustained (sVT).

HF was recognized in the presence of typical symptoms (e.g. breathlessness or fatigue), accompanied by structural and/or functional cardiac abnormalities, resulting in a reduced cardiac output and/or elevated intracardiac pressures. The symptoms of HF were assessed using New York Heart Association classification (NYHA classes 1-4). The HF condition was considered stable when there had been no worsening of $\mathrm{HF}$ for $\geq 3$ months. Peripartum cardiomyopathy was defined by the development of maternal systolic HF late in the pregnancy or early in the postpartum period ${ }^{4}$. When the symptoms of HF 
had acute onset, were refractory to medical therapy and led directly to heart transplantation (HTx) or implantation of left ventricular assist device (LVAD) we used the term fulminant HF. End-stage HF was defined as HTx, LVAD implantation or death caused by HF.

MVA was defined as sudden cardiac death (SCD), cardiopulmonary resuscitation (CPR), or appropriate implantable cardioverter defibrillator (ICD) intervention, i.e. an ICD discharge or anti-tachycardia pacing (ATP) for termination of ventricular fibrillation/VT. Death was classified as sudden if it occurred within 1 hour of the onset of cardiac manifestations, or during sleep (in the absence of previous hemodynamic deterioration), or within 24 hours after the patient was last seen apparently stable clinically. Sudden cardiac arrest (SCA) was defined as occurring within 1 hour of the onset of acute symptoms and reversed by CPR. Relatives included all probands' family members with $T T N$ tv identified as a result of cascade screening, irrespective of the degree of kinship.

A family history of SCD was considered positive if $\geq 1$ first degree relative had died suddenly before the age of 50 years.

\subsection{Biomarkers' measurements}

The plasma levels of NT-proBNP were measured by the electrochemiluminescent immunoassays Elecsys 2010 (Roche, Germany) with the upper limit of normal values defined by the manufacturer at $125 \mathrm{pg} / \mathrm{ml}$. The plasma levels of cardiac troponin T were measured by the troponin T hs-STAT (Roche, Germany) with the upper limit of normal values defined by the manufacturer at $14 \mathrm{ng} / \mathrm{l}$. All measurements were performed in the National Institute of Cardiology laboratory.

\subsection{DNA sequencing and TTN mutation analysis}

DNA was extracted from the peripheral blood by phenol extraction, salting out method or using Genomic Maxi AX kit (A\&A Biotechnology, Gdynia, Poland). Next generation sequencing (NGS) was performed in 46 probands using: whole exome sequencing (WES) in 27 probands, TruSight One Sequencing Panel Kit (TSO) in 4 probands or TruSight Cardio Sequencing Kit (TSC) in 15 probands (Illumina, San Diego, CA, USA). WES libraries were prepared using TruSeq Exome Enrichment Kit (llumina), Nextera DNA Sample Preparation Kits (Illumina), or Twist Human Core Exome Kit (Twist Bioscience, South San Francisco, CA, USA). TSO sequencing was performed similarly to WES using Nextera DNA Sample Preparation Kit (Illumina) with only difference in the enrichment probes used. WES and TSO libraries were paired-end sequenced (2x100 bp) on Illumina HiSeq1500 or NovaSeq 6000 and TSC libraries on Illumina MiSeqDx. Library preparation, sequencing, and data analysis were performed as described previously ${ }^{45}$. TTNtv identified with NGS were followed-up in probands and relatives with Sanger sequencing using BigDye Terminator v3.1 or v1.1 Cycle Sequencing Kit (Life Technologies, Carlsbad, CA, USA) according to the manufacturer's instructions and the $3500 x \mathrm{~L}$ or $3130 \mathrm{xl}$ Genetic Analyzer (Life Technologies). The results were analyzed with Variant Reporter 1.1 Software (Life Technologies). Other genes with strong or moderate evidence of causative relationship with DCM (ACTC1, ACTN2, BAG3, DES, DSP, FLNC, JPH2, LMNA, MYH7, NEXN, PLN, RBM20, SCN5A, TNNC1, TNNI3, TNNT2, TPM1, VCL) ${ }^{46}$ were also inspected for 
presence of rare variants. Identified variants were classified according to ACMG criteria ${ }^{17,47}$. Carriers of pathogenic or likely pathogenic variants in other DCM-related genes were excluded from the study.

\subsection{Statistical analysis}

All results for categorical variables were presented as counts and percentages and for continuous variables as mean and standard deviation (SD) or median and quartiles (Q1:25th, Q2:75th percentiles). The chi-square independence or Fisher exact test was used for comparison of categorical variables. The differences between continuous variables were tested by Student's t-test (for two independent samples, normally distributed data), or in the case of skewed distribution, non-parametric Mann-Whitney tests. A receiver-operating characteristic curve (ROC) analysis was used to assess the cut-off point of the markers for the prediction of events. The optimal cut off was defined as the value with the maximal sum of sensitivity and specificity. Event analysis over time was made by using the univariable and multivariable Cox proportional-hazards regression model. In order to indicate independent predictors of events the stepwise (backward) variable selection procedure was used. All variables with significant prognostic impact in univariate analysis $(p \leq 0.10)$ were included in the multivariable model. Then a backward selection was used to creating the final model. The proportionality of hazards was verified using the weighted Schoenfeld residuals. Model discrimination was assessed using Harrell's Concordance Statistics (C-index). Risk was quantified as a hazard ratio (HR) with 95\% confidence interval (Cl). In case of a zero number of events in one of subgroups, HRs were calculated using the Firth's penalized likelihood approach. Survival curves were constructed by the Kaplan-Meier method and compared by the log-rank test. In subjects without an event, the follow-up period extended to the most recent evaluation or the date of May 31st, 2021. All hypotheses were two-tailed with a 0.05 type I error. All statistical analyses were performed using SAS statistical software, version 9.4 (SAS Institute, Cary, NC, USA).

\subsection{Study limitations}

The major limitation of the study is small sample size due to its single-centre character and hence, small number of major cardiovascular events, precluding the use of multivariate analysis models. The retrospective observational design of the study may include confounders. This study comes from a tertiary referral centre, one of two leading cardiology centers in Poland performing HTx, therefore patients may present with more severe disease than patients usually admitted in other centers.

\section{Declarations}

\section{Author contributions:}

Conceptualization, P.C. and Z.T.B.; data curation, P.C., I.K., G.T.; formal analysis, P.C., and I.K.; investigation, P.C., G.T., E.M., M.S.-M., M.F., P.S., M.S.-W., A.O., M.L., P.L., M.B., T.Z., and Z.T.B.; methodology, P.C., M.R., I.K., R.P., and Z.T.B.; supervision, R.P. and Z.T.B.; writing-original draft, P.C., G.T., I.K., and Z.T.B.; writing-review and editing, P.C., M.R., E.M., M.S.-M., M.F., P.S., M.S.-W., A.O., M.L., P.L., M.B., T.Z., and R.P. All authors have read and agreed to the published version of the manuscript. 


\section{Competing interests.}

The authors declare no competing interests.

\section{References}

1. Hershberger, R. E. \& Morales, A. in GeneReviews(R) (eds M. P. Adam et al.) (University of Washington, Seattle, 1993 [Last Update: September 24, 2015]).

2. Herman, D. S. et al. Truncations of titin causing dilated cardiomyopathy. N Engl J Med, 366, 619-628 https://doi.org/10.1056/NEJMoa1110186 (2012).

3. Franaszczyk, M. et al. Titin Truncating Variants in Dilated Cardiomyopathy - Prevalence and Genotype-Phenotype Correlations. PLoS One, 12, e0169007 https://doi.org/10.1371/journal.pone.0169007 (2017).

4. Ware, J. S. et al. Shared Genetic Predisposition in Peripartum and Dilated Cardiomyopathies. N Engl J Med, 374, 233-241 https://doi.org/10.1056/NEJMoa1505517 (2016).

5. Tayal, U. et al. Phenotype and Clinical Outcomes of Titin Cardiomyopathy. J Am Coll Cardiol, 70, 2264-2274 https://doi.org/10.1016/j.jacc.2017.08.063 (2017).

6. Roberts, A. M. et al. Integrated allelic, transcriptional, and phenomic dissection of the cardiac effects of titin truncations in health and disease. Sci Trans/ Med, 7, 270276 https://doi.org/10.1126/scitranslmed.3010134 (2015).

7. Jansweijer, J. A. et al. Truncating titin mutations are associated with a mild and treatable form of dilated cardiomyopathy. Eur J Heart Fail, 19, 512-521 https://doi.org/10.1002/ejhf.673 (2017).

8. Haggerty, C. M. et al. Genomics-First Evaluation of Heart Disease Associated With Titin-Truncating Variants., 140, 42-54 https://doi.org/10.1161/circulationaha.119.039573 (2019).

9. Kryczka, K. E. et al. Severe Course of Peripartum Cardiomyopathy and Subsequent Recovery in a Patient with a Novel TTN Gene-Truncating Mutation. Am J Case Rep, 19, 820-824 https://doi.org/10.12659/ajcr.909601 (2018).

10. Ware, J. S. et al. Genetic Etiology for Alcohol-Induced Cardiac Toxicity. J Am Coll Cardiol, 71, 22932302 https://doi.org/10.1016/j.jacc.2018.03.462 (2018).

11. Garcia-Pavia, P. et al. Genetic Variants Associated With Cancer Therapy-Induced Cardiomyopathy., 140, 31-41 https://doi.org/10.1161/circulationaha.118.037934 (2019).

12. Felkin, L. E. et al. JAMA Cardiol, Vol. 1, 234-235 (2016).

13. Valverde-Gomez, M. et al. Truncating titin variants in dilated cardiomyopathy: not only LVEF recovery, but also maintenance. Rev Esp Cardiol (Engl Ed), https://doi.org/10.1016/j.rec.2019.12.005 (2020).

14. Tayal, U. et al. Truncating Variants in Titin Independently Predict Early Arrhythmias in Patients With Dilated Cardiomyopathy. J Am Coll Cardiol, 69, 2466-2468 https://doi.org/10.1016/j.jacc.2017.03.530 (2017). 
15. Corden, B. et al. Association of Titin-Truncating Genetic Variants With Life-threatening Cardiac Arrhythmias in Patients With Dilated Cardiomyopathy and Implanted Defibrillators. JAMA Netw Open, 2, e196520 https://doi.org/10.1001/jamanetworkopen.2019.6520 (2019).

16. Akhtar, M. M. et al. Clinical Phenotypes and Prognosis of Dilated Cardiomyopathy Caused by Truncating Variants in the TTN Gene. Circ Heart Fail, 13, e006832 https://doi.org/10.1161/circheartfailure.119.006832 (2020).

17. Richards, S. et al. Standards and guidelines for the interpretation of sequence variants: a joint consensus recommendation of the American College of Medical Genetics and Genomics and the Association for Molecular Pathology. Genet Med, 17, 405-424 https://doi.org/10.1038/gim.2015.30 (2015).

18. Bilinska, Z. T. et al. Left ventricular enlargement is common in relatives of patients with dilated cardiomyopathy. Journal of cardiac failure, 1, 347-353 (1995).

19. Michels, V. V. et al. The frequency of familial dilated cardiomyopathy in a series of patients with idiopathic dilated cardiomyopathy. The New England journal of medicine, 326, 77-82 (1992).

20. Baig, M. K. et al. Familial dilated cardiomyopathy: cardiac abnormalities are common in asymptomatic relatives and may represent early disease. Journal of the American College of Cardiology, 31, 195-201 (1998).

21. Schafer, S. et al. Titin-truncating variants affect heart function in disease cohorts and the general population. Nat Genet, 49, 46-53 https://doi.org/10.1038/ng.3719 (2017).

22. Pinto, Y. M. et al. Proposal for a revised definition of dilated cardiomyopathy, hypokinetic non-dilated cardiomyopathy, and its implications for clinical practice: a position statement of the ESC working group on myocardial and pericardial diseases. Eur Heart J, 37, 1850-1858 https://doi.org/10.1093/eurheartj/ehv727 (2016).

23. Huttner, I. G. et al. A-Band Titin Truncation in Zebrafish Causes Dilated Cardiomyopathy and Hemodynamic Stress Intolerance. Circ Genom Precis Med, 11, e002135 https://doi.org/10.1161/circgen.118.002135 (2018).

24. Pirruccello, J. P. et al. Titin Truncating Variants in Adults Without Known Congestive Heart Failure. J Am Coll Cardiol, 75, 1239-1241 https://doi.org/10.1016/j.jacc.2020.01.013 (2020).

25. McKie, P. M. et al. High-sensitivity troponin I and amino-terminal pro-B-type natriuretic peptide predict heart failure and mortality in the general population. Clin Chem, 60, 1225-1233 https://doi.org/10.1373/clinchem.2014.222778 (2014).

26. Stege, N. M., de Boer, R. A., van den Berg, M. P. \& Silljé, H. H. W. The Time Has Come to Explore Plasma Biomarkers in Genetic Cardiomyopathies. Int J Mol Sci, 22, https://doi.org/10.3390/ijms22062955 (2021).

27. Suthahar, N. et al. Sex-Specific Associations of Cardiovascular Risk Factors and Biomarkers With Incident Heart Failure. J Am Coll Cardiol, 76, 1455-1465 https://doi.org/10.1016/j.jacc.2020.07.044 (2020). 
28. Grzybowski, J. et al. Plasma concentrations of $\mathrm{N}$-terminal atrial natriuretic peptide are raised in asymptomatic relatives of dilated cardiomyopathy patients with left ventricular enlargement., $\mathbf{8 8}$, 191-192 https://doi.org/10.1136/heart.88.2.191 (2002).

29. Chmielewski, P. et al. Can Circulating Cardiac Biomarkers Be Helpful in the Assessment of LMNA Mutation Carriers? J Clin Med, 9, https://doi.org/10.3390/jcm9051443 (2020).

30. Vissing, C. R. et al. Dilated cardiomyopathy caused by truncating titin variants: long-term outcomes, arrhythmias, response to treatment and sex differences. J Med Genet, https://doi.org/10.1136/jmedgenet-2020-107178 (2020).

31. Arbustini, E. et al. Autosomal dominant dilated cardiomyopathy with atrioventricular block: a lamin A/C defect-related disease. Journal of the American College of Cardiology, 39, 981-990 (2002).

32. Nakajima, K. et al. Clinical Manifestations and Long-Term Mortality in Lamin A/C Mutation Carriers From a Japanese Multicenter Registry. Circ J, 82, 2707-2714 https://doi.org/10.1253/circj.CJ-180339 (2018).

33. Kumar, S. et al. Long-Term Arrhythmic and Nonarrhythmic Outcomes of Lamin A/C Mutation Carriers. J Am Coll Cardiol, 68, 2299-2307 https://doi.org/10.1016/j.jacc.2016.08.058 (2016).

34. Saj, M. et al. Variants of the lamin A/C (LMNA) gene in non-valvular atrial fibrillation patients: a possible pathogenic role of the Thr528Met mutation. Mol Diagn Ther, 16, 99-107 https://doi.org/10.1007/bf03256434 (2012).

35. Choi, S. H. et al. Association Between Titin Loss-of-Function Variants and Early-Onset Atrial Fibrillation., 320, 2354-2364 https://doi.org/10.1001/jama.2018.18179 (2018).

36. Ponikowski, P. et al. 2016 ESC Guidelines for the diagnosis and treatment of acute and chronic heart failure: The Task Force for the diagnosis and treatment of acute and chronic heart failure of the European Society of Cardiology (ESC)Developed with the special contribution of the Heart Failure Association (HFA) of the ESC. Eur Heart J 37, 2129-2200, doi:10.1093/eurheartj/ehw128 (2016).

37. Ozmen, C. et al. Association among tenascin-C and NT-proBNP levels and arrhythmia prevalence in heart failure. Clin Invest Med, 40, E219-e227 https://doi.org/10.25011/cim.v40i6.29122 (2017).

38. Medina, A. et al. Brain natriuretic peptide and the risk of ventricular tachyarrhythmias in mildly symptomatic heart failure patients enrolled in MADIT-CRT. Heart Rhythm, 13, 852-859 https://doi.org/10.1016/j.hrthm.2015.12.024 (2016).

39. Patton, K. K. et al. N-terminal pro-B-type natriuretic peptide is associated with sudden cardiac death risk: the Cardiovascular Health Study. Heart Rhythm, 8, 228-233 https://doi.org/10.1016/j.hrthm.2010.10.038 (2011).

40. Verdonschot, J. A. J. et al. Titin cardiomyopathy leads to altered mitochondrial energetics, increased fibrosis and long-term life-threatening arrhythmias. Eur Heart J, 39, 864-873 (2018).

41. Holmström, M. et al. Late gadolinium enhanced cardiovascular magnetic resonance of lamin A/C gene mutation related dilated cardiomyopathy. J Cardiovasc Magn Reson, 13, 30 https://doi.org/10.1186/1532-429x-13-30 (2011). 
42. Giudicessi, J. R., Shrivastava, S., Ackerman, M. J. \& Pereira, N. L. Clinical Impact of Secondary Risk Factors in TTN-Mediated Dilated Cardiomyopathy. Circ Genom Precis Med, 14, e003240 https://doi.org/10.1161/circgen.120.003240 (2021).

43. Bondue, A. et al. Complex roads from genotype to phenotype in dilated cardiomyopathy: scientific update from the Working Group of Myocardial Function of the European Society of Cardiology. Cardiovasc Res, 114, 1287-1303 https://doi.org/10.1093/cvr/cvy122 (2018).

44. Akhtar, M. \& Elliott, P. M. Risk Stratification for Sudden Cardiac Death in Non-Ischaemic Dilated Cardiomyopathy. Curr Cardiol Rep, 21, 155 https://doi.org/10.1007/s11886-019-1236-3 (2019).

45. Ploski, R. et al. Does p.Q247X in TRIM63 cause human hypertrophic cardiomyopathy? Circ Res, 114, e2-5 https://doi.org/10.1161/CIRCRESAHA.114.302662 (2014).

46. Jordan, E. et al. An Evidence-Based Assessment of Genes in Dilated Cardiomyopathy., https://doi.org/10.1161/circulationaha.120.053033 (2021).

47. Tavtigian, S. V. et al. Modeling the ACMG/AMP variant classification guidelines as a Bayesian classification framework. Genet Med, 20, 1054-1060 https://doi.org/10.1038/gim.2017.210 (2018).

\section{Figures}

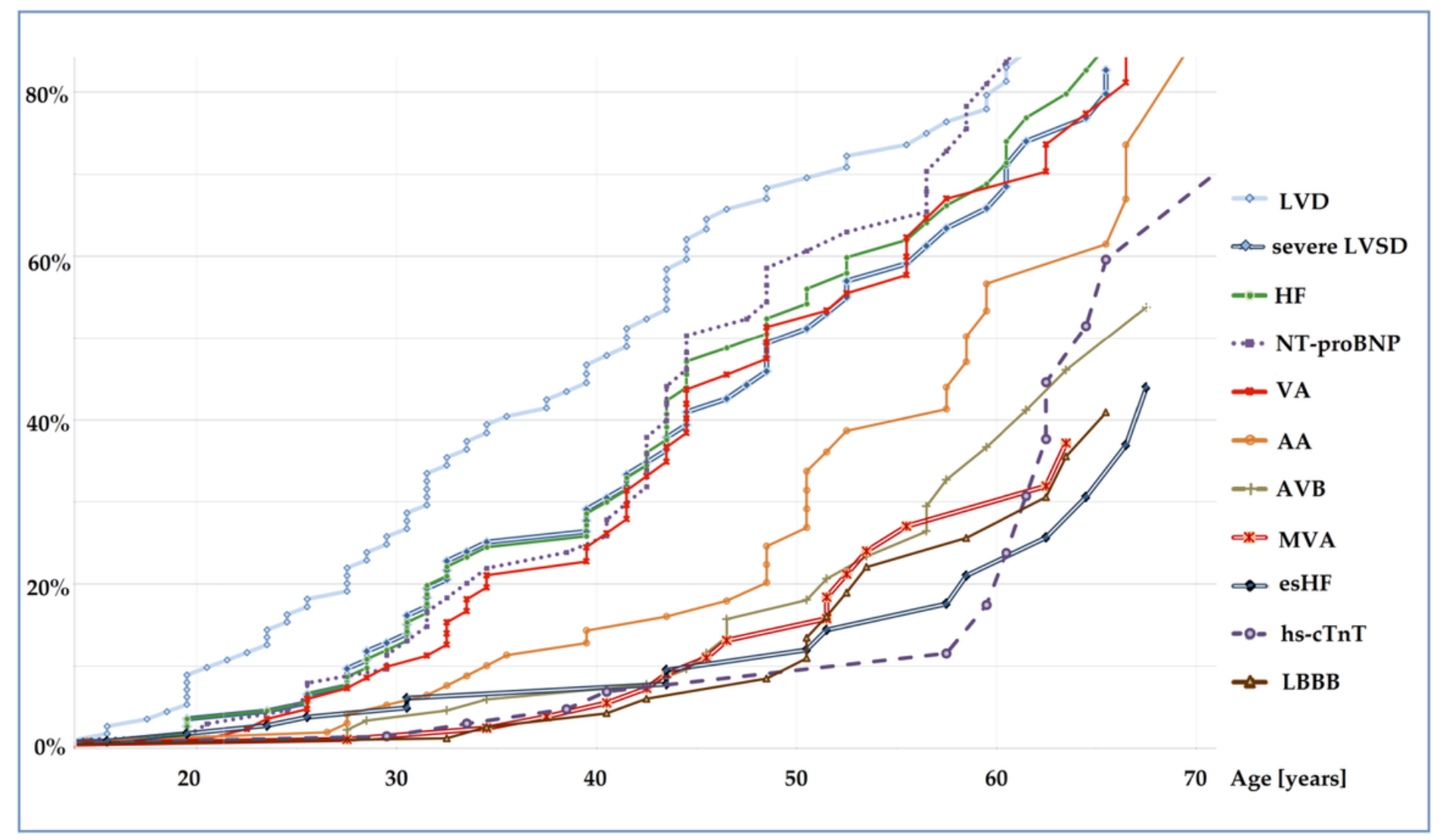

Figure 1 
Penetrance of disease symptoms in the whole study cohort of TTNtv carriers. Legend: AA, atrial arrhythmia; AVB, atrioventricular block; esHF, end-stage heart failure; HF, heart failure; hs-cTnT, highsensitivity cardiac troponin T concentration >14 ng/l; LBBB, left bundle branch block; LVD, left ventricular dysfunction; severe LVSD, severe left ventricular systolic dysfunction; MVA, malignant ventricular arrhythmia; NT-proBNP, N-terminal pro-B-type natriuretic peptide serum concentration $>125 \mathrm{pg} / \mathrm{ml}$; TTNtv, titin truncating variants; $V A$, ventricular arrhythmia.

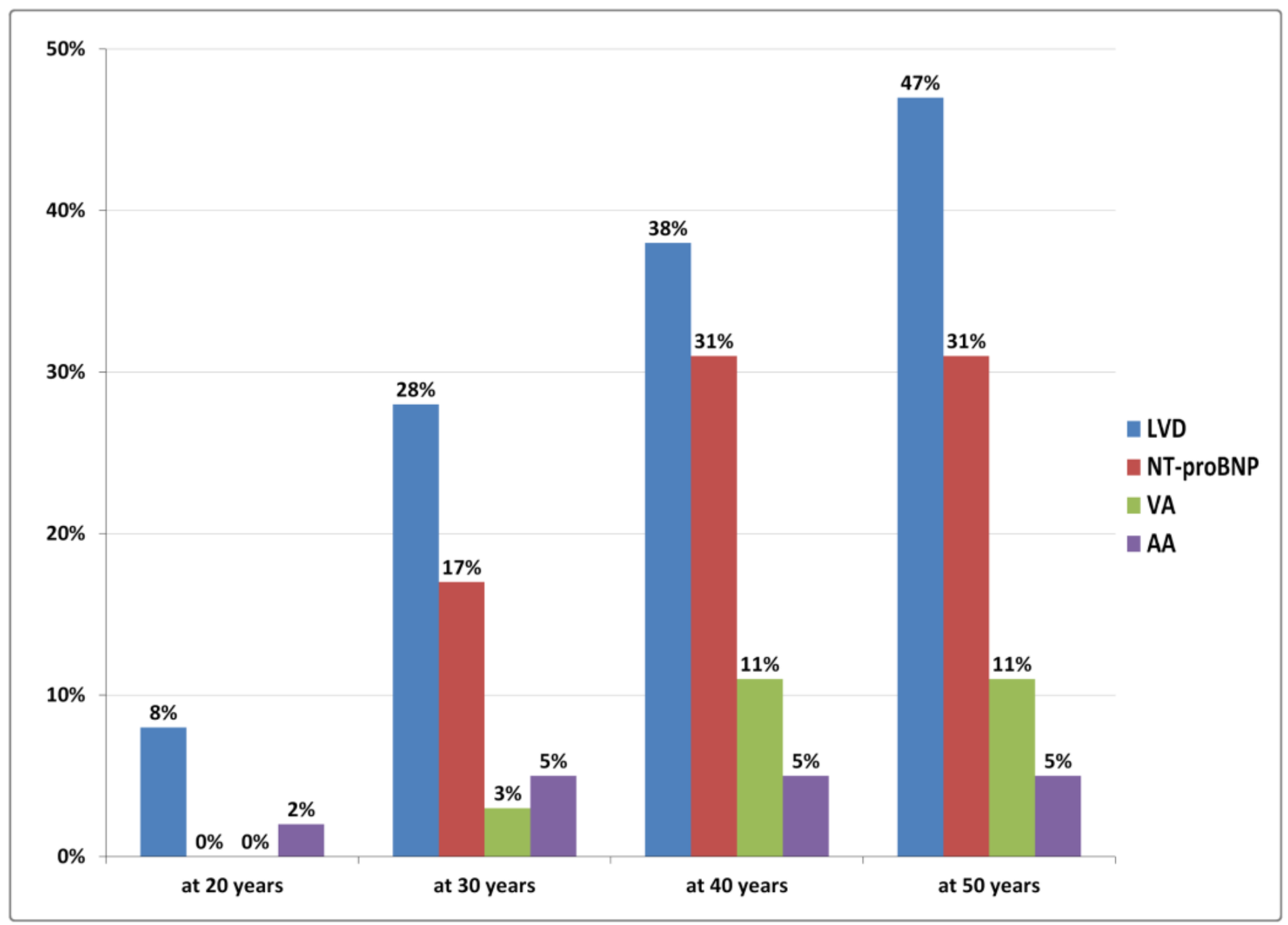

Figure 2

Penetrance of cardiotitinopathy indicators in asymptomatic TTNtv carriers identified through familial screening. Legend: AA, atrial arrhythmia; LVD, left ventricular dysfunction, NT-proBNP, N-terminal pro-Btype natriuretic peptide serum concentration $>125 \mathrm{pg} / \mathrm{ml}$; VA, ventricular arrhythmia. 


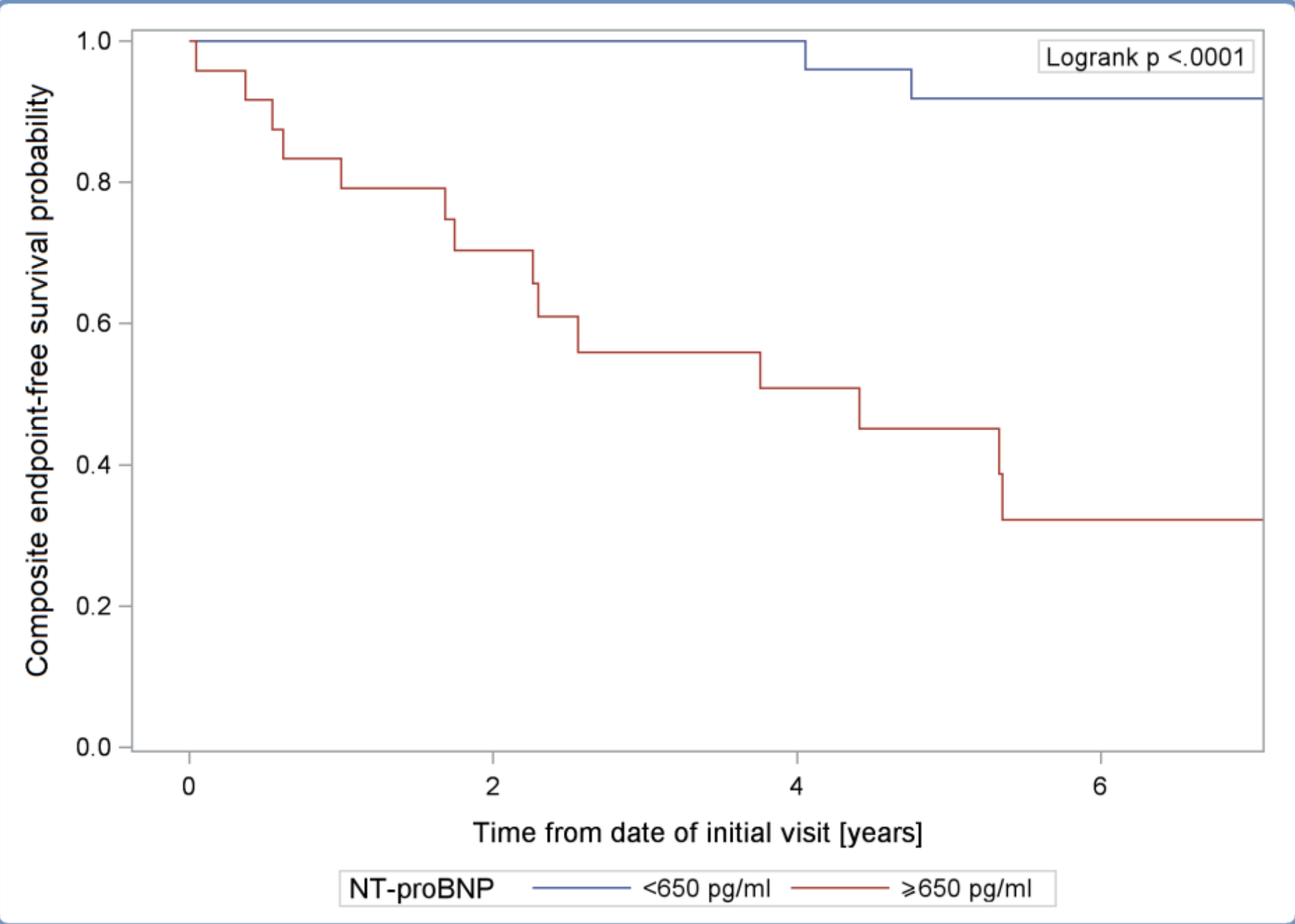

\section{Figure 3}

Kaplan-Meier composite endpoint-free survival curves in cardiotitinopathy according to NT-proBNP serum concentration. Legend: NT-proBNP, N-terminal pro-B-type natriuretic peptide serum concentration. 


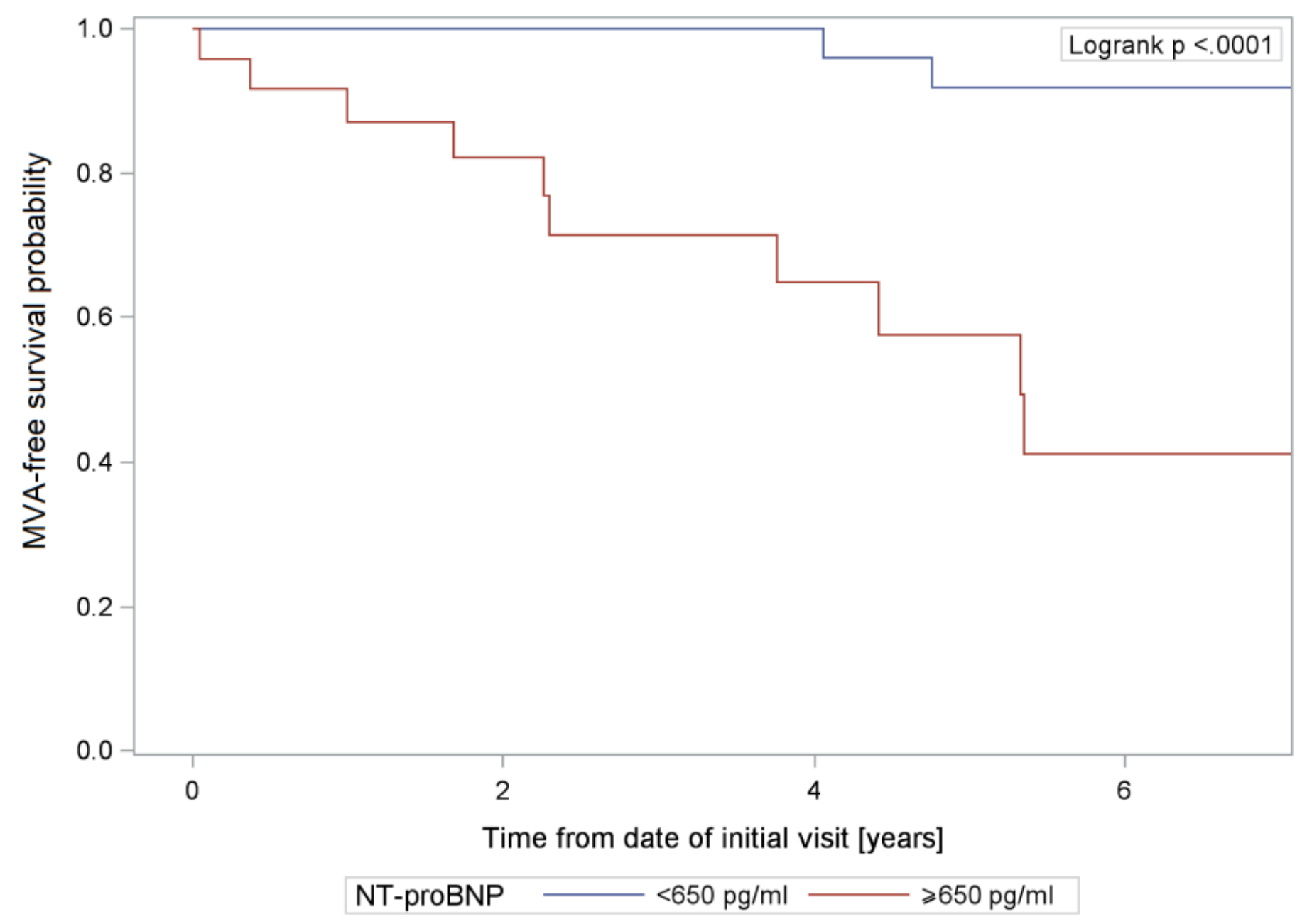

\section{Figure 4}

Kaplan-Meier malignant ventricular arrhythmia-free survival curves in cardiotitinopathy according to NTproBNP serum concentration. Legend: MVA, malignant ventricular arrhythmia; NT-proBNP, N-terminal proB-type natriuretic peptide serum concentration.

\section{Supplementary Files}

This is a list of supplementary files associated with this preprint. Click to download.

- TTNrelatedSupplementarytables.docx 